\title{
Impact of White Matter Lesions on Depression in the Patients with Alzheimer's Disease
}

\author{
Jung Jae Lee', Eun Young Lee', Seok Bum Lee', Joon Hyuk Park², Tae Hui Kim³, \\ Hyun-Ghang Jeong ${ }^{4}$, Jae Hyoung $\mathrm{Kim}^{5}$, Ji Won $\mathrm{Han}^{6}$, and Ki Woong Kim${ }^{6,7,8} \bowtie$ \\ 'Department of Psychiatry, Dankook University College of Medicine, Cheonan, Republic of Korea \\ 2Department of Psychiatry, Jeju National University School of Medicine, Jeju National University Hospital, Jeju, Republic of Korea \\ ${ }^{3}$ Department of Psychiatry, Yonsei University Wonju Severance Christian Hospital, Wonju, Republic of Korea \\ ${ }^{4}$ Department of Psychiatry, Korea University Guro Hospital, Korea University College of Medicine, Seoul, Republic of Korea \\ ${ }^{5}$ Department of Radiology, Seoul National University Bundang Hospital, Seongnam, Republic of Korea \\ ${ }^{6}$ Department of Neuropsychiatry, Seoul National University Bundang Hospital, Seongnam, Republic of Korea \\ ${ }^{7}$ Department of Psychiatry, Seoul National University College of Medicine, Seoul, Republic of Korea \\ ${ }^{8}$ Department of Brain and Cognitive Science, Seoul National University College of Natural Sciences, Seoul, Republic of Korea
}

Objective Comorbid depression is common in patients with Alzheimer's disease (AD). An increase in white matter lesions (WMLs) has been associated with depression in both elderly individuals with normal cognition and patients with Alzheimer's disease. We investigated whether the severity and location of WMLs influence the association between WMLs and comorbid depression in AD.

Methods We enrolled $93 \mathrm{AD}$ patients from Seoul National University Bundang Hospital. We administered both the Mini International Neuropsychiatric Inventory (MINI) and the Korean version of the Consortium to Establish a Registry for Alzheimer's Disease Assessment Packet (CERAD-K) clinical and neuropsychological battery. Subjects also underwent brain magnetic resonance imaging (MRI). We diagnosed $\mathrm{AD}$ according to the criteria of the National Institute of Neurological and Communicative Disorders and Stroke and the Alzheimer's Disease and Related Disorders Association. We diagnosed depressive disorders according to the DSM-IV diagnostic criteria, and evaluated the severity of depressive symptoms using the Korean version of the Geriatric Depression Scale (GDS-K). We quantified the WML volumes from the brain MRI using a fully automated segmentation algorithm.

Results The log of the WML volume in the frontal lobe was significantly associated with depressive disorders (odds ratio=1.905, 95\% $\mathrm{CI}=1.027-3.533, \mathrm{p}=0.041$ ), but not with the severity of depressive symptoms as measured by the GDS-K.

Conclusion The WML volume in the frontal lobe conferred a risk of comorbid depressive disorders in AD, which implies that comorbid depression in $\mathrm{AD}$ may be attributed to vascular causes.

Psychiatry Investig 2015;12(4):516-522

Key Words Alzheimer's disease, White matter lesions, Depression.

\section{INTRODUCTION}

White matter lesions (WMLs) are commonly found in the T2-weighted magnetic resonance imaging (MRI) or fluid-attenuated inversion recovery (FLAIR) scans of normal elderly people. ${ }^{1}$ WMLs also have been found in Alzheimer's disease $(\mathrm{AD}),{ }^{2}$ vascular dementia, ${ }^{3,4}$ and depression, ${ }^{5-7}$ and have been

Received: April 14, 2015 Revised: May 16, 2015

Accepted: May 16, 2015 Available online: September 30, 2015

$\triangle$ Correspondence: Ki Woong Kim, MD, PhD

Department of Neuropsychiatry, Seoul National University Bundang Hospital, 166 Gumi-ro, Bundang-gu, Seongnam 463-707, Republic of Korea

Tel: +82-31-787-7432, Fax: +82-31-787-4058, E-mail: kwkimmd@snu.ac.kr

(a) This is an Open Access article distributed under the terms of the Creative Commons Attribution Non-Commercial License (http://creativecommons.org/licenses/by$\mathrm{nc} / 3.0$ ) which permits unrestricted non-commercial use, distribution, and reproduction in any medium, provided the original work is properly cited. associated with vascular risk factors such as hypertension and diabetes. ${ }^{8,9}$ Neuropathologically, WMLs are characterized by the demyelination of white matter axons, mild fibrillary gliosis, and fibrohyaline thickening of small vessels that supply the white matter. ${ }^{10,11}$ In particular, irregular periventricular WMLs and deep WMLs have been associated with microcystic infarcts and patchy rarefaction of the myelin, which are ischemic in nature. ${ }^{12,13}$ These findings support a prominent role of vascular factors in the pathogenesis of WMLs.

Pathological evidence suggests that WMLs may play a role in the clinical symptoms of AD. Individuals with more WMLs have a higher risk of developing AD. ${ }^{14,15}$ In addition, WMLs are associated with cognitive dysfunction and the severity of the dementia. ${ }^{16,17}$ However, the relationship between depression and WMLs in AD remains unclear. Several studies have 
explored the relationship between WMLs and depression. WMLs, particularly in the frontal area, have been found to be associated with higher depression scores..$^{18,20}$ Other studies, however, did not find such a relationship. ${ }^{21-23}$ The heterogeneity of the study populations, the methods by which depression was assessed, and the measurements of the WML volumes might contribute to these inconsistent results. In most studies, different semi-quantitative visual rating scales, such as Fazeka's scale and Scheltons's scale, were used. ${ }^{18,19,21-23}$ In one study, an automated volumetric method was used to assess WML volumes, but depression was assessed by rating scales rather than by clinical diagnosis. ${ }^{20}$ In other studies, depression was assessed by clinical diagnosis, but WMLs were assessed by visual rating scales. ${ }^{21,22}$

The present study avoided some of the limitations of these previous studies by 1) using automated quantitative volumetric methods to assess the severity and location of WMLs; 2) using both a structured clinical interview and a self-report questionnaire to diagnose the presence and severity of depression; and 3) recruiting only $\mathrm{AD}$ patients to avoid a confounding effect from a heterogeneous study population.

The purpose of this study was to explore the relationship between depression and the severity and location of WMLs in patients with $\mathrm{AD}$. We tested the hypothesis that larger WML volumes and frontal WMLs in particular are associated with depressive mood and clinician-diagnosed depression in patients with $\mathrm{AD}$.

\section{METHODS}

\section{Subjects}

We recruited $\mathrm{AD}$ patients from a dementia clinic (Seoul National University Bundang Hospital, SNUBH) and communities [the Korean Longitudinal Study on Health and Aging $(\mathrm{KLoSHA})^{24}$ and the nationwide dementia screening and registry program (DSRP)]. All subjects underwent standardized clinical interviews and neurological and physical examinations, which were administered by four geriatric psychiatrists with advanced training in neuropsychiatry and dementia research according to the protocol of the Korean version of the Consortium to Establish a Registry for Alzheimer's Disease clinical assessment battery (CERAD-K), ${ }^{25}$ the standardized TIA/stroke form from the Atherosclerosis Risk in Communities Study (ARIC) ${ }^{26}$ and the Mini International Neuropsychiatric Interview (MINI), ${ }^{27}$ to diagnose dementia, stroke, and major psychiatric disorders, respectively.

Dementia was diagnosed in these participants in accordance with the Diagnostic and Statistical Manual of Mental Disorders, Fourth Edition (DSM-IV) criteria, ${ }^{28}$ and probable or possible $\mathrm{AD}$ was diagnosed according to the National Insti- tute of Neurological and Communicative Disorders and Stroke and the Alzheimer's Disease and Related Disorders Association (NINCDS-ADRDA) criteria. ${ }^{29} \mathrm{~A}$ total of 93 subjects met the inclusion criteria. Sixty-six (71\%) subjects had probable $\mathrm{AD}$ and 27 (29\%) had possible AD.

All the participants were fully informed regarding their study participation and either the participant or a legal guardian provided written informed consent. The study protocol was approved by the Institutional Review Board of SNUBH.

\section{Assessment of depression}

Depression was evaluated based on information provided from the patients as well as information provided by their primary caregiver. First, we administered a self-report questionnaire, the Korean version of the Geriatric Depression Scale (GDS-K), to assess subjective depressive mood..$^{30}$ Second, research-trained psychiatrists interviewed the patients and(or) primary caregivers using the MINI to clinically diagnose depression. ${ }^{27}$ The severity of depression was rated from very mild (subsyndromal depression) to moderate-to-severe depression (e.g., minor and major depressive disorder, dysthymic disorder).

Major depressive disorder (MDD) and dysthymic disorder were diagnosed according to the DSM-IV criteria, and minor depressive disorder $(\mathrm{MnDD})$ was diagnosed according to the research criteria proposed in the DSM-IV. Subsyndromal depression (SSD) was diagnosed according to the following operational criteria: 1) two or more symptoms listed in the DSMIV criterion A for a major depressive episode occurring during the same two-week period; 2) at least one symptom should be depressed mood or anhedonia; 3) each depressive symptom should be present for more than half of one day or more than seven days during the 2 weeks; and 4) symptoms do not meet the criteria for a diagnosis of MDD or MnDD. Subjects were divided into two diagnostic group: 1) Depression, which was defined as evidence of MDD, MnDD, dysthymic disorder, or SSD or 2) No depression, which was defined as no current evidence of significant depressed mood.

\section{Assessment of neuropsychological functions}

Global cognitive function was evaluated using the Mini-Mental Status Examination (MMSE) ${ }^{25,31}$ To assess memory function, the word list memory test (WLMT), the word list recall test (WLRT), and the word list recognition test (WLRcT) from the Korean version of the CERAD Neuropsychological Assessment Battery (CERAD-K-N) were administered. Language function was evaluated using the verbal fluency test (VFT) and the 15-item modified Boston Naming Test (mBNT) from the CERAD-K-N. ${ }^{25,31}$ Visuospatial function was evaluated using the constructional praxis test (CPT) from the CERAD-K-N. ${ }^{25,31}$ Frontal function was evaluated using the trail making test-A/ 
B (TMT-A/B) from the CERAD-K-N. ${ }^{25,31}$ All the assessments were performed at SNUBH

\section{Assessment of WMLs}

T1-weighted MR and FLAIR images were acquired on a 3.0 Tesla MRI scanner system (Achieva, Philips Medical System, The Netherlands) at SNUBH. The T1 images were acquired using the following protocol: magnetic field $=3.0$ Tesla; sequence $=S P G R$; repetition time $/$ echo time $=22 / 4 \mathrm{msec}$; number of excitations $=1$; flip angle $=40^{\circ}$; field of view $=240$ $\mathrm{mm}$; plane $=$ sagittal; matrix $=256 \times 256 \mathrm{~mm}$; thickness $=1 \mathrm{~mm}$; inter-slice gap $=$ none; slice number $=120$. The FLAIR images were acquired using the following protocol: magnetic field $=3.0$ Tesla; repetition time/echo time $=9900 / 160 \mathrm{msec}$; number of excitations $=1$; flip angle $=90^{\circ}$; field of view $=240 \mathrm{~mm}$; axial plane matrix $=256 \times 256 \mathrm{~mm}$; thickness $=3 \mathrm{~mm}$; and inter-slice gap $=$ none.

All the processes were implemented in Matlab 2010a (The MathWorks, Inc., Natick, MA, USA). WMLs were automatically segmented from the FLAIR images according to the method adopted by Yoo et al. ${ }^{32} \mathrm{~A}$ study-specific T1 template (SST) was constructed for accurate spatial normalization using the DARTEL toolbox in SPM8 (Wellcome Trust Centre for Neuroimaging, London, UK; http://www.fil.ion.ucl.ac.uk/ spm). All the T1 images, as well as the FLAIR and segmented WML images, were spatially transformed to the SST.

To define the regions to be classified as WMLs, a mask-labeled region was manually delineated from the MNI standard space by one neurological expert. Frontal, temporal, parietal, and occipital WML regions were defined. The MNI152 standard T1 image as well as the mask-labeled region was spatially transformed to the SST. Sub-classified WML images were obtained by applying the transformed mask to the segmented WML images through voxel-wise image multiplication for each individual WMH image. The sub-classified WMH volumes then were computed by counting the voxels from the labeled WML images.

\section{Statistical analysis}

We computed descriptive statistics on the demographic variables and disease-related measures using independent $\mathrm{t}$-tests for each continuous variable and chi-square tests for each categorical variable to examine the differences between the groups. The neuropsychological measures between the groups were compared by an analysis of variance with adjustments for age, education, and GDS-K scores, which are well-established risk factors for cognitive dysfunction. ${ }^{31,33,34}$ We used a logarithmic transformation to produce normally distributed WML volumes. The associations between the log-transformed WML volumes and clinical variables were examined using
Pearson's correlation coefficient or Spearman's rank-order correlation coefficient (r) as appropriate. We also performed multivariate logistic regression analyses. First, we conducted unadjusted analyses examining the relationship between log WML volumes and the dependent variables. Second, we included two sets of adjustment variables: 1) those characteristics well-known to influence depression, i.e., age, gender, and level of education ${ }^{35,36}$ and 2) vascular risk factors (e.g., cardiovascular disease, hypertension, diabetes) ${ }^{37}$ and prior depression history. ${ }^{38,39}$ Due to the high correlation between the total WML volume and regional WML volumes, separate models were generated to analyze the relationship between depression and WML volume by location. For all the analyses, a twotailed p-value of less than 0.05 was considered statistically significant. All the analyses were conducted using SPSS version 12.0 (SPSS Inc., Chicago, IL, USA).

\section{RESULTS}

The clinical and demographic characteristics of the participants are summarized in Table 1. Of the 93 subjects, 25 were diagnosed with depression and 68 were assigned to the no depression group. No differences between the groups were observed on any clinical or demographic variables except GDS-K scores. The neuropsychological performance of the participants is summarized in Table 2. No differences were observed between the depression and no depression groups.

We performed multiple logistic regressions to assess the relationship between clinician-diagnosed depression and the total and regional WML volumes with adjustments for potential confounding effects of vascular risk factors and residual clinical variables. The total log WML volume was not significantly associated with the presence of clinician-diagnosed depression (odds ratio $1.820,95 \%$ CI $0.962-3.443, \mathrm{p}=0.066$ ). When the WML volumes were analyzed separately with respect to location, the log frontal WML volume was significantly associated with the presence of clinician-diagnosed depression (odds ratio 1.905, 95\% CI=1.027-3.533, $\mathrm{p}=0.041$ ), but no significant relationship was observed in any other WML region (Table 3).

Table 4 shows the relationship between the GDS-K scores as well as other clinical variables and the log total or regional WML volumes. The GDS-K scores were not associated with either the log total WML volume ( $\mathrm{p}=0.369$, Pearson's chisquared test) or the log frontal WML volume ( $\mathrm{p}=0.410$, Pearson's chi-squared test).

\section{DISCUSSION}

Depression has been recognized as one of the most fre- 
Table 1. Sociodemographic characteristics of the study subjects

\begin{tabular}{lccc}
\hline & Depression $(\mathrm{N}=25)$ & No depression $(\mathrm{N}=68)$ & p-value* \\
\hline Age (years), mean \pm SD & $76.12 \pm 7.61$ & $77.63 \pm 7.09$ & 0.373 \\
Female, N (\%) & $18(72.0)$ & $49(72.1)$ & 0.996 \\
Education (years), mean \pm SD & $8.16 \pm 6.14$ & $7.93 \pm 5.60$ & 0.863 \\
CDR-SOB & $3.94 \pm 2.79$ & $4.45 \pm 3.30$ & 0.464 \\
GDS-K, mean \pm SD & $17.56 \pm 6.25$ & $11.07 \pm 5.26$ & $<0.001$ \\
Cardiovascular disease, N (\%) & $2(8.0)$ & $7(10.3)$ & 0.740 \\
Hypertension, N (\%) & $13(52.0)$ & $36(52.9)$ & 0.936 \\
Diabetes, N (\%) & $6(24.0)$ & $14(20.6)$ & 0.723 \\
Head trauma, N (\%) & $3(12.0)$ & $6(8.8)$ & 0.646 \\
Kidney disease, N (\%) & $0(0.0)$ & $4(5.9)$ & 0.215 \\
Prior depression, N (\%) & $7(28.0)$ & $17(25.0)$ & 0.769 \\
\hline
\end{tabular}

${ }^{*}$-tests for continuous variables and chi-square tests for categorical variables. GDS-K: Korean version of the Geriatric Depression Scale, CDRSOB: Clinical Dementia Rating Scale-Sum of Boxes

Table 2. Neurocognitive function of the participants according to their depression diagnosis

\begin{tabular}{lccc}
\hline & $\begin{array}{c}\text { Depression } \\
\mathrm{N}=25\end{array}$ & $\begin{array}{c}\text { No depression } \\
\mathrm{N}=68\end{array}$ & p-value* \\
\hline Word Fluency & $8.3 \pm 3.2$ & $8.4 \pm 3.7$ & 0.787 \\
Boston Naming & $8.0 \pm 3.0$ & $7.7 \pm 3.2$ & 0.892 \\
MMSE-KC & $17.9 \pm 5.1$ & $16.6 \pm 5.5$ & 0.348 \\
Word List Memory & $9.3 \pm 3.0$ & $8.5 \pm 4.0$ & 0.491 \\
Constructional Praxis & $7.7 \pm 2.5$ & $8.1 \pm 2.3$ & 0.304 \\
Word Delayed Recall & $1.6 \pm 1.5$ & $1.2 \pm 1.6$ & 0.429 \\
Word Recognition & $5.0 \pm 3.2$ & $4.5 \pm 2.8$ & 0.502 \\
Constructional Recall & $1.3 \pm 1.7$ & $1.3 \pm 2.1$ & 0.747 \\
Trail Making A & $173.7 \pm 106.4$ & $171.0 \pm 111.6$ & 0.455 \\
Trail Making B & $280.2 \pm 34.2$ & $277.2 \pm 53.4$ & 0.296 \\
\hline
\end{tabular}

*ANOVA adjusted for age, sex, and education. MMSE-KC: Korean version of the Mini-Mental Status Examination

Table 3. Multiple logistic regression analysis of the association between clinician-diagnosed depression and the log of the white matter lesion volume

\begin{tabular}{llcc}
\hline & Log WML & Odds ratio $(95 \%$ CI $)$ & p-value \\
\hline Model 1 & Total & $1.820(0.962-3.443)$ & 0.066 \\
Model 2 & Frontal & $1.905(1.027-3.533)$ & 0.041 \\
Model 3 & Temporal & $1.611(0.962-2.699)$ & 0.070 \\
Model 4 & Parietal & $1.146(0.850-1.544)$ & 0.797 \\
Model 5 & Occipital & $1.092(0.754-1.581)$ & 0.643 \\
\hline
\end{tabular}

Each model adjusted for age, sex, education, cardiovascular disease, hypertension, diabetes, and prior depression. WML: white matter lesion volume $(\mathrm{mL})$

quent psychiatric comorbidities of $\mathrm{AD}$, and previous studies have suggested that $20 \%$ to $50 \%$ of patients with $\mathrm{AD}$ suffer from depression. ${ }^{40-42}$ The importance of depression in $\mathrm{AD}$ is underscored by its negative impact on the patient's quality of life, ${ }^{43}$ cognitive ability, ${ }^{44}$ and activities of daily living ${ }^{45}$ as well as the caregiver's stress and psychological comorbidity. ${ }^{46}$ Shortterm mortality in elderly subjects with less severe dementia
Table 4. Correlations between the Geriatric Depression Scale and the log white matter lesion volume and other sociodemographic factors

\begin{tabular}{lccc}
\hline \multirow{2}{*}{ Factors } & \multicolumn{2}{c}{ Correlation coefficient } & p-value \\
\cline { 2 - 3 } & Pearson $(\mathrm{r})$ & Spearman $(\rho)$ & \\
\hline Age & -0.099 & & 0.346 \\
Gender & -0.124 & & 0.235 \\
Education & -0.174 & & 0.096 \\
MMSE-KC & -0.170 & & 0.112 \\
Cardiovascular disease & & 0.018 & 0.862 \\
Hypertension & & -0.161 & 0.124 \\
Diabetes & & 0.068 & 0.515 \\
Prior depression & & 0.259 & 0.012 \\
Log WML & & & \\
$\quad$ Total & 0.094 & & 0.369 \\
Frontal & 0.086 & & 0.410 \\
Temporal & 0.072 & & 0.495 \\
Parietal & 0.034 & & 0.749 \\
Occipital & 0.091 & & 0.387 \\
\hline
\end{tabular}

WML: white matter lesion volume (mL), MMSE-KC: Korean version of the Mini-Mental Status Examination

is predicted by the presence of subsyndromal depression and mood symptoms. ${ }^{47}$ Identifying and treating patients with depression in $\mathrm{AD}$ may reverse this associated morbidity.

The etiologies and pathophysiology of depression in $\mathrm{AD}$ remains unclear. Depression can be organic in nature due to cerebrovascular changes. ${ }^{5,48}$ The neurodegenerative process may directly contribute to the development of depression. Several studies have found that depression has been associated with the loss of noradrenergic or serotonergic nuclei ${ }^{49,50}$ or as a consequence of low-grade inflammation in the brain. ${ }^{51}$

In our study, the WML volume, especially the frontal WML volume, was positively and independently correlated with depression after adjusting for vascular risk factors. This result is consistent with some previous studies. ${ }^{18-20}$ The WMLs are a 
surrogate marker of small-vessel vascular disease resulting from ischemic damage due to chronic hypoperfusion, whereas mild periventricular WMLs are non-ischemic in origin. Recently, Brickman et al..$^{52}$ reported that reduced cerebral autoregulation was associated with an increased WML volume. There is growing evidence that frontal and subcortical WMLs are implicated in late-life depression and influence the outcome, possibly via the disruption of frontal-subcortical circuits. ${ }^{2,33,54}$ These findings, together with those in the present study, suggest that vascular depression can be considered an etiology of depression in AD. WMLs may affect mood symptoms via the disruption of intracerebral connectivity, compromising efficient neuronal communication. The prefrontal cortex and its extensive cortical-cortical and cortical-subcortical connectivity is thought to play a central role in motivation-based depressive symptoms in dementia. ${ }^{55}$

The results from previous studies have been inconsistent with respect to the relationship between WMLs and depression in $\mathrm{AD}$. Several factors may contribute to these inconsistencies.

First, different visual rating scales were used across studies, ${ }^{18,19,21-23}$ and therefore scores from different rating scales are not directly comparable. Different visual rating scales can produce inconsistent results among neuroimaging studies. ${ }^{56}$ Furthermore, visual rating scales are less sensitive than volumetric methods. ${ }^{57}$ Soennesyn et al. ${ }^{20}$ used both automated volumetric WML measurement and a visual rating scale, and reported that the automated volumetric measure of the total and frontal WMLs but not the visual rating scale scores were associated with the severity of depressive symptoms. This finding suggests that visual rating scales inevitably have some limitations such as data nonlinearity, lack of sensitivity to small changes, and susceptibility to ceiling effects. ${ }^{8,58,59}$

Second, the methods to assess depression were different across studies. Diagnosing depression in AD remains problematic for several reasons. First, the symptoms of depression in $\mathrm{AD}$ overlap with other neuropsychiatric manifestations of dementia. The use of rating scales in a patient with dementia without consideration of this overlap in nonspecific depressive symptoms may result in an erroneous assignment of a depression diagnosis in $\mathrm{AD} .{ }^{60}$ Second, another complicating factor in the diagnosis of depression in $\mathrm{AD}$ is the patient's limited ability to communicate distress coherently. Patients with dementia cannot always accurately report their own symptoms, and therefore may underreport their symptoms. ${ }^{61} \mathrm{AD}$ patients may also perceive themselves as less depressed than do their caregivers or a clinician. ${ }^{62}$ Third, depressive symptoms in patients with $\mathrm{AD}$ differ from those in depressed but otherwise cognitively normal elderly patients. Patients with $\mathrm{AD}$ appear to have more motivational symptoms and delusions and less commonly experience sadness, sleep disturbance, and appetite loss. ${ }^{41,63,64}$ Fourth, there are currently no standardized methods to diagnose depression in $\mathrm{AD}$. The recommendation for diagnosing depression in $\mathrm{AD}$ is to use a structured psychiatric interview; depression scales should be used to rate the severity of the depression but not to diagnose. In our study, we used both a self-rating depression questionnaire and a structured psychiatric interview to assess depression. Interestingly, we found a significant association between clinician-diagnosed depression and WML volumes but failed to show such a relationship between self-reported depression scores and WML volumes.

The heterogeneity of the study populations may also contribute to the inconsistent results across studies. Some studies included only patients with $\mathrm{AD},{ }^{21,22}$ but other studies included patients with other types of dementia such as vascular dementia, dementia with Lewy bodies, and frontotemporal dementia as well as $\mathrm{AD} \cdot{ }^{18-20,23}$

The prevention or reduction of WMHs could lessen the burden of depression and depressive symptoms in patients with dementia. The presence of WMLs, in particular in the frontal area, is associated with depression in $\mathrm{AD}$, which implies a common pathophysiology of depression caused by disruption to the frontal-subcortical circuits. Understanding the pathogenesis is important and may offer important insights into therapeutic options for the treatment and prevention of depression in $\mathrm{AD}$.

To our knowledge, this is the first study to investigate the association between depression and WMLs in AD using a standardized structured psychiatric interview to assess depression and an automated quantitative measurement to assess the severity and location of WMLs. However, this study was crosssectional in design and the depression group was relatively small, which may warrant a prospective study on a larger sample to confirm the positive correlation between WMLs in AD and depression and investigate its clinical significance.

\section{Acknowledgments}

This study was supported by grant no. 04-2008-001 from the SNUBH Research Fund and by a grant from the Korean Health Technology R\&D Project, Ministry of Health and Welfare, Republic of Korea [grant number HI09C1379 (A092077)].

\section{REFERENCES}

1. Schmidt R, Fazekas F, Offenbacher H, Dusek T, Zach E, Reinhart B, et al. Neuropsychologic correlates of MRI white matter hyperintensities: a study of 150 normal volunteers. Neurology 1993;43:2490-2494.

2. O’Brien J, Desmond P, Ames D, Schweitzer I, Harrigan S, Tress B. A magnetic resonance imaging study of white matter lesions in depression and Alzheimer's disease. Br J Psychiatry 1996;168:477-485.

3. Almkvist O, Wahlund LO, Andersson-Lundman G, Basun H, Backman L. White-matter hyperintensity and neuropsychological functions in dementia and healthy aging. Arch Neurol 1992;49:626-632. 
4. Schmidt R. Comparison of magnetic resonance imaging in Alzheimer's disease, vascular dementia and normal aging. Eur Neurol 1992;32:164169.

5. Alexopoulos GS, Meyers BS, Young RC, Campbell S, Silbersweig D, Charlson M. 'Vascular depression' hypothesis. Arch Gen Psychiatry 1997;54:915-922.

6. Krishnan KR, Hays JC, Blazer DG. MRI-defined vascular depression. Am J Psychiatry 1997;154:497-501.

7. Lesser IM, Hill-Gutierrez E, Miller BL, Boone KB. Late-onset depression with white matter lesions. Psychosomatics 1993;34:364-367.

8. Longstreth WT Jr, Manolio TA, Arnold A, Burke GL, Bryan N, Jungreis $\mathrm{CA}$, et al. Clinical correlates of white matter findings on cranial magnetic resonance imaging of 3301 elderly people. The Cardiovascular Health Study. Stroke 1996;27:1274-1282.

9. de Leeuw FE, de Groot JC, Oudkerk M, Witteman JC, Hofman A, van Gijn J, et al. Hypertension and cerebral white matter lesions in a prospective cohort study. Brain 2002;125:765-772.

10. Englund E. Neuropathology of white matter changes in Alzheimer's disease and vascular dementia. Dement Geriatr Cogn Disord 1998;9(Suppl 1):6-12.

11. Ogata J. Vascular dementia: the role of changes in the vessels. Alzheimer Dis Assoc Disord 1999;13(Suppl 3):S55-S58.

12. Thomas AJ, O'Brien JT, Barber R, McMeekin W, Perry R. A neuropathological study of periventricular white matter hyperintensities in major depression. J Affect Disord 2003;76:49-54.

13. van Swieten JC, van den Hout JH, van Ketel BA, Hijdra A, Wokke JH van Gijn J. Periventricular lesions in the white matter on magnetic resonance imaging in the elderly. A morphometric correlation with arteriolosclerosis and dilated perivascular spaces. Brain 1991;114(Pt 2):761774 .

14. Prins ND, van Dijk EJ, den Heijer T, Vermeer SE, Koudstaal PJ, Oudkerk $\mathrm{M}$, et al. Cerebral white matter lesions and the risk of dementia. Arch Neurol 2004;61:1531-1534.

15. Vermeer SE, Prins ND, den Heijer T, Hofman A, Koudstaal PJ, Breteler MM. Silent brain infarcts and the risk of dementia and cognitive decline. N Engl J Med 2003;348:1215-1222.

16. Stout JC, Jernigan TL, Archibald SL, Salmon DP. Association of dementia severity with cortical gray matter and abnormal white matter volumes in dementia of the Alzheimer type. Arch Neurol 1996;53:742-749.

17. Diaz JF, Merskey H, Hachinski VC, Lee DH, Boniferro M, Wong CJ, et al. Improved recognition of leukoaraiosis and cognitive impairment in Alzheimer's disease. Arch Neurol 1991;48:1022-1025.

18. Barber R, Scheltens P, Gholkar A, Ballard C, McKeith I, Ince P, et al. White matter lesions on magnetic resonance imaging in dementia with Lewy bodies, Alzheimer's disease, vascular dementia, and normal aging. J Neurol Neurosurg Psychiatry 1999;67:66-72.

19. O'Brien J, Perry R, Barber R, Gholkar A, Thomas A. The association between white matter lesions on magnetic resonance imaging and noncognitive symptoms. Ann N Y Acad Sci 2000;903:482-489.

20. Soennesyn H, Oppedal K, Greve OJ, Fritze F, Auestad BH, Nore SP, et al. White matter hyperintensities and the course of depressive symptoms in elderly people with mild dementia. Dement Geriatr Cogn Dis Extra 2012;2:97-111.

21. Clark LM, McDonald WM, Welsh-Bohmer KA, Siegler IC, Dawson DV, Tupler LA, et al. Magnetic resonance imaging correlates of depression in early- and late-onset Alzheimer's disease. Biol Psychiatry 1998;44: 592-599.

22. Harrell LE, Duvall E, Folks DG, Duke L, Bartolucci A, Conboy T, et al. The relationship of high-intensity signals on magnetic resonance images to cognitive and psychiatric state in Alzheimer's disease. Arch Neurol 1991;48:1136-1140.

23. Lind K, Jonsson M, Karlsson I, Sjogren M, Wallin A, Edman A. Depressive symptoms and white matter changes in patients with dementia. Int J Geriatr Psychiatry 2006;21:119-125.

24. Park JH, Lim S, Lim JY, Han MK, Yoon IY, Kim JM, et al. An overview of the Korean longitudinal study on health and aging. Psychiatry Investig 2007;4:84-95.

25. Lee JH, Lee KU, Lee DY, Kim KW, Jhoo JH, Kim JH, et al. Development of the Korean version of the Consortium to Establish a Registry for Alzheimer's Disease Assessment Packet (CERAD-K): clinical and neuropsychological assessment batteries. J Gerontol B Psychol Sci Soc Sci 2002;57:47-53.

26. The Atherosclerosis Risk in Communities (ARIC) Study: design and objectives. The ARIC investigators. Am J Epidemiol 1989;129:687-702.

27. Yoo SW, Kim YS, Noh JS, Oh KS, Kim CH, Namkoong K. Validity of Korean version of the Mini-International Neruopsychiatric Interview. Anxiety Mood 2006;2:50-55.

28. Americn Psychiatric Association. Diagnostic and Statistical Manual of Mental Disorders, Fourth Edition Revised (DSM-IV). Washington, DC: American Psychiatric Association; 1994.

29. McKhann G, Drachman D, Folstein M, Katzman R, Price D, Stadlan EM. Clinical diagnosis of Alzheimer's disease: report of the NINCDSADRDA Work Group under the auspices of Department of Health and Human Services Task Force on Alzheimer's Disease. Neurology 1984;34:939-944.

30. Cho MJ, Bae JN, Suh GH, Hahm BJ, Kim JK, Lee DW, et al. Validation of Geriatric Depression Scale, Korean Version (GDS) in the Assessment of DSM-III-R Major depression. J Korean Neuropsychiatr Assoc 1999;31:48-63.

31. Lee DY, Lee KU, Lee JH, Kim KW, Jhoo JH, Kim SY, et al. A normative study of the CERAD neuropsychological assessment battery in the Korean elderly. J Int Neuropsychol Soc 2004;10:72-81.

32. Yoo BI, Lee JJ, Han JW, Oh SY, Lee EY, MacFall JR, et al. Application of variable threshold intensity to segmentation for white matter hyperintensities in fluid attenuated inversion recovery magnetic resonance images. Neuroradiology 2014;56:265-281.

33. Lichtenberg PA, Ross T, Millis SR, Manning CA. The relationship between depression and cognition in older adults: a cross-validation study. J Gerontol B Psychol Sci Soc Sci 1995;50:25-32.

34. Wilson RS, Barnes LL, Mendes de Leon CF, Aggarwal NT, Schneider JS, Bach J, et al. Depressive symptoms, cognitive decline, and risk of AD in older persons. Neurology 2002;59:364-370.

35. Park JH, Kim KW, Kim MH, Kim MD, Kim BJ, Kim SK, et al. A nationwide survey on the prevalence and risk factors of late life depression in South Korea. J Affect Disord 2012;138:34-40.

36. Park JH, Lee JJ, Lee SB, Huh Y, Choi EA, Youn JC, et al. Prevalence of major depressive disorder and minor depressive disorder in an elderly Korean population: results from the Korean Longitudinal Study on Health and Aging (KLoSHA). J Affect Disord 2010;125:234-240.

37. Djernes JK. Prevalence and predictors of depression in populations of elderly: a review. Acta Psychiatr Scand 2006;113:372-387.

38. Alexopoulos GS. Depression in the elderly. Lancet 2005;365:19611970.

39. Teodorczuk A, O’Brien JT, Firbank MJ, Pantoni L, Poggesi A, Erkinjuntti $\mathrm{T}$, et al. White matter changes and late-life depressive symptoms: longitudinal study. Br J Psychiatry 2007;191:212-217.

40. Lyketsos CG, Steinberg M, Tschanz JT, Norton MC, Steffens DC, Breitner JC. Mental and behavioral disturbances in dementia: findings from the Cache County Study on Memory in Aging. Am J Psychiatry 2000;157:708-714.

41. Olin JT, Katz IR, Meyers BS, Schneider LS, Lebowitz BD. Provisional diagnostic criteria for depression of Alzheimer disease: rationale and background. Am J Geriatr Psychiatry 2002;10:129-141.

42. Olin JT, Schneider LS, Katz IR, Meyers BS, Alexopoulos GS, Breitner JC, et al. Provisional diagnostic criteria for depression of Alzheimer disease. Am J Geriatr Psychiatry 2002;10:125-128.

43. Gonzalez-Salvador T, Lyketsos CG, Baker A, Hovanec L, Roques C, Brandt J, et al. Quality of life in dementia patients in long-term care. Int J Geriatr Psychiatry 2000;15:181-189.

44. Bassuk SS, Berkman LF, Wypij D. Depressive symptomatology and in- 
cident cognitive decline in an elderly community sample. Arch Gen Psychiatry 1998;55:1073-1081.

45. Lyketsos CG, Steele C, Baker L, Galik E, Kopunek S, Steinberg M, et al. Major and minor depression in Alzheimer's disease: prevalence and impact. J Neuropsychiatry Clin Neurosci 1997;9:556-561.

46. Gonzalez-Salvador MT, Arango C, Lyketsos CG, Barba AC. The stress and psychological morbidity of the Alzheimer patient caregiver. Int J Geriatr Psychiatry 1999;14:701-710.

47. Janzing JG, Bouwens JM, Teunisse RJ, Van't Hof MA, Zitman FG. The relationship between depression and mortality in elderly subjects with less severe dementia. Psychol Med 1999;29:979-983.

48. O'Brien JT, Ames D. White matter lesions in depression and Alzheimer's disease. Br J Psychiatry 1996;169:671.

49. Forstl H, Burns A, Luthert P, Cairns N, Lantos P, Levy R. Clinical and neuropathological correlates of depression in Alzheimer's disease. Psychol Med 1992;22:877-884.

50. Zweig RM, Ross CA, Hedreen JC, Steele C, Cardillo JE, Whitehouse $\mathrm{PJ}$, et al. The neuropathology of aminergic nuclei in Alzheimer's disease. Ann Neurol 1988;24:233-242.

51. Leonard BE. Inflammation, depression and dementia: are they connected? Neurochem Res 2007;32:1749-1756.

52. Brickman AM, Guzman VA, Gonzalez-Castellon M, Razlighi Q, Gu Y, Narkhede A, et al. Cerebral autoregulation, beta amyloid, and white matter hyperintensities are interrelated. Neurosci Lett 2015;592:54-58.

53. Hickie I, Scott E, Wilhelm K, Brodaty H. Subcortical hyperintensities on magnetic resonance imaging in patients with severe depression--a longitudinal evaluation. Biol Psychiatry 1997;42:367-374.

54. Greenwald BS, Kramer-Ginsberg E, Krishnan KR, Ashtari M, Auerbach C, Patel M. Neuroanatomic localization of magnetic resonance imaging signal hyperintensities in geriatric depression. Stroke 1998;29: 613-617.

55. Naarding P, de Koning I, van Kooten F, Janzing JG, Beekman AT, Koudstaal PJ. Post-stroke dementia and depression: frontosubcortical dys- function as missing link? Int J Geriatr Psychiatry 2007;22:1-8

56. Mantyla R, Erkinjuntti T, Salonen O, Aronen HJ, Peltonen T, Pohjasvaara $\mathrm{T}$, et al. Variable agreement between visual rating scales for white matter hyperintensities on MRI. Comparison of 13 rating scales in a poststroke cohort. Stroke 1997;28:1614-1623.

57. van Straaten EC, Fazekas F, Rostrup E, Scheltens P, Schmidt R, Pantoni L, et al. Impact of white matter hyperintensities scoring method on correlations with clinical data: the LADIS study. Stroke 2006;37:836-840.

58. Fazekas F, Chawluk JB, Alavi A, Hurtig HI, Zimmerman RA. MR signal abnormalities at $1.5 \mathrm{~T}$ in Alzheimer's dementia and normal aging. AJR Am J Roentgenol 1987;149:351-356.

59. Melhem ER, Herskovits EH, Karli-Oguz K, Golay X, Hammoud DA, Fortman BJ, et al. Defining thresholds for changes in size of simulated T2-hyperintense brain lesions on the basis of qualitative comparisons. AJR Am J Roentgenol 2003;180:65-69.

60. Burke WJ, Rubin EH, Morris JC, Berg L. Symptoms of "depression" in dementia of the Alzheimer type. Alzheimer Dis Assoc Disord 1988;2: 356-362.

61. Burke WJ, Roccaforte WH, Wengel SP, McArthur-Miller D, Folks DG, Potter JF. Disagreement in the reporting of depressive symptoms between patients with dementia of the Alzheimer type and their collateral sources. Am J Geriatr Psychiatry 1998;6:308-319.

62. Teri L, Wagner AW. Assessment of depression in patients with Alzheimer's disease: concordance among informants. Psychol Aging 1991; 6:280-285.

63. Janzing JG, Hooijer C, van 't Hof MA, Zitman FG. Depression in subjects with and without dementia: a comparison using GMS-AGECAT. Int J Geriatr Psychiatry 2002;17:1-5.

64. Zubenko GS, Zubenko WN, McPherson S, Spoor E, Marin DB, Farlow $\mathrm{MR}$, et al. A collaborative study of the emergence and clinical features of the major depressive syndrome of Alzheimer's disease. Am J Psychiatry 2003;160:857-866. 